\title{
Inhalt der ersten Hälfte des Bandes XXVI. (1870) der Fortschritte der Physik.
}

\author{
Erster Abschnitt: Allgemeine Physik.
}

1. Maass und Messen: Englischer Comitébericht 3. - L. Levi 4. - Morin 4. - v. Steinheil's Comparator 5. - Martins 6. - Soleil 7. - Maclear 7. H. Wild 8. - W. Förster 10. - Tafeln zur Ermittelung der Gewichtszulagen 13. Tabellen für Raummasse 15. - Baeyer 16. - C. W. Baur 17. - Hirsch und Plantamour 18. - W. Jordan 18. - Th. Albrecbt 19. - Lyman 20. - Williamson 20. - G. Schweizer 21. - v. Villarceau 21. - Starke 22. - d'Abbadie 22. I3. Wolf 23. - d'Abbadie 23. - v. Villarceau 24. - Hoüel 25. - v. Villarceau 25. - d'Abbadie 25. - v. Villarceau 26. - Bunge's Wage 27. - Havos 28. - Duckham's Wage 29. - Westphal 29. - Cotton und Pilcher 30. - S. Short 31: - K. L. Baver 31. - Hhipson 33. - Arzberger 34. - Lagout 35. - Nolan's Distanzmesser 3.̆. - Lohmeier's Beobachtungen 36. - Zemann 37. - Tennant 38. Ellery 38 - Bellanger's Curvigraph 39. - Crofton 39. - Wolf 40. - Zöllner 40. - Litteratur 41.

2. Diehtigkeit: Topsöe 42. - Metz 43. -- Schweikert 43. -- v. Baumhaver 44. - A. Horstmann 44. - A. Naumann 44. - de Negri 45. - Wurtz 45. Hossetti 45. - Wolffenstein 46. - Soret 46. - Thomsen 46. - Bardeleben 47. A. Bauer 47. - J. Bayer 47. - Litteratur 47.

3. Molekularphysik: 0. Popp 48. - Hofmann 49. - Schlösing 49. Sidot 50. - Zöppriz 50. - Pfaunder 51. - Isambert 52. - Lamy 53. II. Devlle 54. - Stein 57. -. Dilte 57. - - Debray 57. - Mobr 58. - Gunoing 59. - W. Müller 60. - J. Dewar 61. - W. Thomson 62. - Ladenburg 63. Mendelejeff 63. - Groshans 63. - Blomstrand 64. - L. Meyer 64. - Muck 65. H. Deville 65. - Krebs 66. - Blondlot 66. - Schultz-Sellak 67. - R. Weber 67. - Bettendorff u. v. Rath 68. - Rathke 68. - Morren 68. - Baumbauer 69. Lewald 69. - Rammelsberg 70. - Grotb 71. - Rose 73. - Jannettaz 73. Credner 74. - Hinrichs 75. - Hinrichs 75. - Litteratur 76.

4. Mechanik: de Mondésir 80. - Krumme 81. - Preece 81. - Walton 81. - Walton 82. - Ferrers 82. - Tait 82. - Leray 83. - Morin 83. - Tresca 83. - de St.-Venant 83. - de St.-Venant 84. - Boussinesq 84. - C. Neumann 85. Whitworth 85. - Noble 85. — Darapsky 85. - de Brettes 86. - Faye 87. Walton 87. - Lecoq de Boisbaudran 88. - Leray u. Lecoq de Boisbaudran 88. H. de la Goupillière 88. - Mousson 89. - Most 89. - Emsmann 89. - Stebnitzki 89. - C. Neumann 90. - "Boileau 91. - Coste 91. - de St.-Venant 91. Lucas 91 v. 92. - Heger 93. - Lowrie 93. - Proctor 93. - Stone 94. Puiseux 94. - Newcomb 93. -- Flammarion 95. - Quesneville 95. - Heppel 96. Rankine 96. - Dupré 96. - Rolland 96. - Most 97. - Tilly 97. - Röntgen 97. - Reusch 98. - J. Müller 98. - J. Grossmann 98. - Litteratur 98.

5. Ifdrodynamik: Kirchboff 100.101. - Clebsch 103. - Kostka 104. Eberbardt 106. - Cockle 106. - Tait 107. - de St.-Venant 107. - de St.-Venant 108. - Boussinesq 110. - Roumiantzof 112. - Challis 112. - Abbgt 114. Garbett 114. - Kutter 115. - Boussinesq 116. - Perrigault 117. - Leclert 118. - Litteratur 118. - "Hasler 119. - Chameroy's Wassermesser 119. - Wither's Wassermesser 120. - Greyveldinger 120. - Boutelon u. Piau 120. - Winkler 121. Bunsen's Wasserluftpumpe 121. - Lagillardais' Heberpumpe 122. - Cooke 122. Vivian 123. - Bleckrod 123. - Antoine 124. - "Dupuis 124. - Tangye's bydraulische Maschine 125. - Ramsbottom's Pumpe 125. - Ackermann 126. Rühlmann 126. - Nagel u. Kämp 127. - Clerk 128. - Perrigault 129. v. Caligny 129. - Comitébericht 130. - Froude 131-132. - Teicbmann 133. Schmitt 133. - Graeff 134. - Litteratur 135.

Fortscbr. d. Phys. XXVI. 
6. Aërodynamik: Rühlmann 137. - Cailletet 138. - Amagat 138. P. d Mondésir 139. - Huber 139. - Dumas 139. - Lavoisier 139. - Meusnier 140. - Hachette 140. - Dapny de Lone 140. - Giffard 142. - Sorel 142. Bouvet 142. - Joulie 143. - Villeneuve 143. - Dumas 143. - Litteratar 143.

7. Cohăsion und Adhăsion. A. Festigkeit und Elasticität: J. King 146. - Desgoffe u. Ollivier 146. - Montucci 146. - H. Maogon u. Tresca 146. Gruner, Boussingault, Fairbairn 147. - Bessemerfabrikate 147. - Schneebeli 149. - A. Wöbler 150. - Tresca, de St.-Venant 150. - Levy 151. - Clark, Jarolimek 154. - Kohlrausch u. Loomis 155. - Frănkel 157. - Boussinesq 157. - Viollet 158. - Leras 158. - v. Reiche 159. - Litteratur 159.

B. Capilla rităt: Becquerel 162 . - จ. d. Mensbrugghe 165. - Lüdtge 166. - v. d. Mensbruggbe 168 . - Duclaux 170. - Limouzin 177. - C. A. Yalson 177. - Luvioi 182. - Marangoni u. Stefanelli 184. - Böttger 186. - Tomlinson 187. - Melde 187. - Sang 188. - Duncan u. Gamgee 189. - H. Quincke 189. Marangoni 191. - Dupré u. Page 196. - Litteratur 196. - Stahl 197. - Boltzmano 199. - P. du Bois-Reymond 200. - Warburg 201. - Moutier 203.

C. Löslichbeit: Lefebrre 203. - Debray 204. - Pohl 204. - Kissel 204. - Struve, Schulz-Sellack 204. - Berthelot 205. - Nichols 205. - Landrin 206. Rosenstiebl, Trémaux 206. - Rosenstiehl u. Rühlmann 207. - Tomlinson 207. Grenfell 208. - Tomlinson 210. - v. Lang 210. - Loschmidt 211. - Wretsehko 213. - Litteratur 214.

D. Absorption: Caron u. H. Deville 216. - Hunter 216. - Roseoe 217. H. Müller 217. - Schober 217. - E. Richters 218. - Seely 218. - O. Loew 219. - Litteratur 219.

E. Adbäsion.

\section{Zweiter Abschnitt: Akustik.}

9. Physikallsehe Akustik: Seebeck 223. - Melde 226. - Warburg 228. Schellbach 230. - Guthrie 23I. - Sondbauss 231. - Strutt 239. - Hoppe 211. -.. J. J. Müller 246. - Töpler u. Boltzmann 248. - Mattbiessen 259. - A. Heller 264. - J. Bourget 266. - F. André 268. - R. König 269. - Cl. Neumann 271. - A. Weiohold 275. - J. Stefan 275. - Litteratur 277.

9. Physiologische Akustlk 280.

Dritter Abschnitt: Optik.

10. Theorie des Liehts: H. Hudson 283. - Flammarion 284. - Cb. Brooke 284. - C. Puschl 286. - Bösser 291. - Litteratur 295.

11. Portpflanzung, Splegelung und Brechung des Liehts: Ricour 296. Gibbs 296. - Veltmann 297. - Klinkerfues 301. - Ketteler 302. - v. Lang 310. - Wernicke 312. - Gladstone 315. - Groullebois 317. - Groullebois, Jamin, Cornu 319. - Croullebois 320. - v. Obermayer 320. - v. d. Willigen 321. Tomlinson 322. - Gibbs 322. - Gibbs 323. - Christiansen 323. - Graffweg 324. - Montigny 326. - A. W. Hofmann 327. - Litteratur 328.

12. Objektive Farben, Spektrum, Absorption: Rayet 329. - Rayet 330. Wolf u. Rayet 330. - Glan 331. - Lippich 332. - Huggins 334. - Davis 335. Secchi 335. - Secchi 336. - Lockyer 338. - Secchi, Fizeau 340. - Lockyer 341. - Reitlinger u. Kubn 342. - Wülloer 344. - Faye 345. - Dubrunfaut 345. Dubrunfaut 345. - Watts 346. - Lecoq de Boisbaudran 346. - Lecoq de Boisbaudran 347. - Cappel 347. - Silliman 350. - Roscoe 350. - Watts 351. Spektrum der Bessemerflamme 351. - Hinrichs 351. - Young 351. - Browning 352. Kundt 352. - Luck 353. - Schultz-Sellack 353. - Vierordt 354. - Janssen 354. Scboras 355. - Sorby 356. - Hagenbach 357. - Aodrews 358. - Ray Lankaster 359. - J. Müller 359. -- Perkin 359. - Sorby 360. -- Litteratur 360.

13. Photometrie: Nagant 366. - Vierordt 367. - Roscoe u. Thorpe 368. v. Bezold 369. - 0. N. Rood 370. -- Rood 370. - W. Crookes 371. Litteratur 372. 
14. Phosphorescenz und FJuorescenz: E. Becquerel 374. - Sarasin 376. de la Rive 376. - Morren 376. - "Secchi 376. - W. Müller 376. - Goppelsröder 377. - "Hagenbach 377. - Hagenbach 377. - "Parnell 378. - Wartba 378. A. Gruner 379. - Moffat 379. - Litteratur 379.

15. Interferenz, Polarisation, Doppelbrechung, Krystalloptik: Soret 380. -Hagenbach 380. - Soret 381. - Lallemand 381. - Tyndall 381. - Hayes 382. - de la Rive 382. - Ditscheiner 382. - Brezina 384. - v. Lang 385. - Pape 387. - des Cloizeaux 388. -- des Cloizeaux 389. - Kosmann 390. - Ditscheiner 391. - Kurz 392. - Litteratur 393.

a. Circularposition: de la Rive 394. - Jellet 396. - Tuchschmid 396. Litteratur 398.

16. Chemische Wirknngen des Llehts: Roscoe u. Thorpe 399. - Morren 399. Tyndall 399. - Lallemand 400. - A. Vogel u. Raab 400. - Loew 400. - Strett u. Franz 400. - Berlandt 401. - Schoras 401. - Böttger 401. - Schnauss 402. - Grüne 403. - Spiller 403. - Woodward 403. - Larrey 403. - Carey Lea 403. - Bazin 404. - H. Vogel 404. - "Déhérain 40 i. - "Loew 404. - "Loew 405. - Tyndall 405. - Prillieux 405. - Roze 405. Bert 405. - Kraus 406. Prillieux 407. - Batalin 408. - Litteratur 408.

18. Physiolngische Optlk: Schneller 409. -- Dobrowsky \$10. - Tennant 4 II. - Tupper 411. - H. Gerold 411. - J. J. Müller 412. - Woinow 412. Bow 412. - Marangoni 413. - Ketteler 414. - Maxwell 414. - L. Hermann 415. K. Exner 415. - J. K. Becker 415. - Dvorák 415. - J. Müller 416. - Tait 416. - S. Exner 417. - Hellgelb die sichtbarste Farbe 418. - Towne 418. - Koblrausch 418. - Woinow 418. - Listing 419. - Steinhauser 419. - Litteratur 420.

18. Optische Apparate: Ad. Martin 422. -- Jouglet 422. - Ackermann 423. A. le Sueur 423. - Ellery \$23. - Robinson 0. Grubb 424. - J. Stoney 424. -J. Stoney 425. - Wecker u. Roger 425. - Simons, Davidson 425. - Cook, Newall's Teleskop 426. - S. B. Kincaid 426. - A. Cazin 427. - Listing 427. - Merz 428. C. A. Young 428. - Smyth 429. - Th. Cooke 429. - S. B. Kincaid 429. Dawes 429. - Listing 430. - Royston-Pigolt 430. - J. H. B. L. 431. - Bäbler 431. - Girard 143. - J. Browning 432. - S. Merz 433. - Merz 433. Schubring 433. - Riefer 434. - Melde 434. - Mejerstein 434. - Steinheil 435. Burow 436. - Laussedat 436. - Feil 437. - Cornu 437. - Litteratur 438.

Vierter Abschnitt: Wärme.

19. Theorie der Wărme: Budde 140 o. s. w.

Da mehreren Herren das Verzeichniss der Druckfehler und Berichtigungen zur ersten Halfte des vorigen Jabrganges, das mit dem vorläufigen Inbaltsverzeichniss gleichzeitig gedruckt war, nicht zogegangen ist, so folgt dasselbe am Schlusse des Bandes.

Durch ein Verseben sind in den früheren Bänden 1867, 1868, 1869 die Bandzahlen für die Reports der British Association unrichtig angegeten. Es baben die Berichte der Meetings von Nottingham 1866, Dundee 1867, und Norwich 1868 die respektiven Bandzablen XXXVI., XXXVII., XXXVIII. Auch sind die beiden Halften der Bull. d. J. soc. chim. 1869, die indess meist nicht nach der Bandzabl, sondern nach den Hälften des Jahrgangs citirt sind, nicht die Bănde XII. u. XIII., sondern XI. u. XII. 